\title{
Prospective validation of the BISHE score as a predictor of the need for massive transfusion in patients with upper gastrointestinal bleeding
}

\section{Yi-Chuan Chen}

Chang Gung Memorial Hospital Chiayi Branch https://orcid.org/0000-0001-6324-561X

\section{Kai-Siang Wu}

Chiayi Chang Gung Memorial Hospital

Ming-Szu Hung (D m12049@adm.cgmh.org.tw)

Chang Gung Memorial Hospital Chiayi Branch

\section{Research article}

Keywords: risk score, scoring system, upper gastrointestinal bleeding, massive transfusion, high-risk

Posted Date: August 11th, 2020

DOI: https://doi.org/10.21203/rs.3.rs-56098/v1

License: (c) (i) This work is licensed under a Creative Commons Attribution 4.0 International License.

Read Full License 


\section{Abstract}

Background: Emergency transfusion strategies for patients with upper gastrointestinal bleeding (UGIB) have not been well developed. The BISHE is a recently developed scoring system for prediction of patients likely to require massive transfusion but has not been validated. In this study, we evaluated the predictive ability of the BISHE score and that of a revised scoring system in an effort to improve care for patients with UGIB and allow better resource allocation.

Methods: This prospective observational cohort study included patients who were admitted to our hospital between July 1, 2016 and June 30, 2019 and required massive transfusion (defined as receiving three units of red blood cells over one hour) in the emergency department. The predictive accuracy of the BISHE score and that of the revised scoring system were compared using area under the receiveroperating characteristic (AUROC) curve analysis.

Results: Liver cirrhosis, an international normalized ratio $>1.5$, and hemoglobin $<8.0 \mathrm{~g} / \mathrm{dL}$ were independently associated $(p<0.05)$ with requirement for massive transfusion. The revised scoring system discriminated successfully between patients with UGIB requiring massive transfusion and their counterparts who did not (AUROC $0.82,95 \%$ confidence interval 0.78-0.86) and performed better than the BISHE score (AUROC 0.73, 95\% confidence interval 0.69-0.76; $p=0.004$ ).

Conclusions: Compared with the BISHE score, the revised scoring system was better able to identify patients likely to require massive transfusion.

\section{Background}

Upper gastrointestinal bleeding (UGIB) is a common indication for blood transfusion and accounts for about $9 \%-21 \%$ of all blood products transfused $(1,2)$. Mortality in life-threatening UGIB is approximately $15 \%(3,4)$. To improve hemostasis and restore oxygen delivery, massive transfusion (MT) for exsanguinating UGIB is considered life-saving $(5,6)$. After surgery and trauma, UGIB is one of the most common causes of MT(7). A previous report indicated that about $5.7 \%$ of all patients with UGIB required MT; however, one recent study reported that about $3 \%$ of patients required transfusion of more than 10 units of red blood cells within 24 hours of $\operatorname{UGIB}(8,9)$. Early initiation of an MT protocol has been associated with a reduction in the overall number of blood products transfused, organ failure, and mortality in patients with trauma(10-12). Several appropriate scoring systems for initiation of an MT protocol have been developed for early and accurate identification of the need for MT(13-15). However, accurate scoring systems that can be used to predict the need for MT in patients with UGIB are limited because those available have been developed mainly in trauma settings and their appropriateness for use in patients with UGIB is unclear.

One study found that the BISHE scoring system had good performance for prediction of the need for MT in patients with UGIB(9). The BISHE score is composed of five clinical elements that can predict the need for MT, including band cells in blood, the international normalized ratio, shock, a hemoglobin level $<8.0$ 
$\mathrm{g} / \mathrm{dL}$, and endoscopic treatment. To calculate the BISHE score, a patient needs to undergo endoscopy to see if the cause of UGIB needs to be treated endoscopically or if it can be treated pharmacologically with a proton pump inhibitor. Therefore, the BISHE score has limited applicability because it can only be used when endoscopy is performed. However, in most clinical circumstances, MT is administered before the patient undergoes endoscopy.

The aims of this study were to validate the BISHE scoring system in patients with UGIB and develop a new scoring method to improve early determination of the need for MT in the emergency department.

\section{Methods}

\section{Study population}

This prospective cohort study was performed at a university-affiliated teaching hospital in central Taiwan that includes an emergency department (ED) with approximately 80,000 patient visits per year. All adult patients (over 18 years of age) who were admitted to the ED at Chiayi Chang Gung Memorial Hospital between July 1, 2016 and June 30, 2019 with evidence of UGIB were eligible for inclusion in the study. Diagnoses of UGIB included hematemesis, melena, bloody or coffee ground vomitus, and bloody or coffee ground material in nasogastric aspirate. The study was approved by Chang Gung Medical Foundation Institutional Review Board (IRB No: 201600157B0). Informed consent was obtained from all study participants. Patients who were unable to provide informed consent were excluded.

\section{Data collection}

Demographic data, including age, sex, vital signs on triage in the ED, chief complaints, present illness, laboratory test results, comorbid medical conditions, current medications, and types of treatment (for example, blood transfusion, therapeutic procedures, operations, and medications) were recorded.

\section{Definitions}

Massive transfusion was defined as transfusion of three or more units of red blood cells within one hour during the ED stay $(16,17)$. Therapeutic procedures were defined as procedures to control or stop bleeding, including therapeutic endoscopy, angiographic embolization, and surgery. Shock was defined as a systolic blood pressure $<100 \mathrm{mmHg}$ at the time of ED triage. Further bleeding was defined as any of the following: (1) repeated esophagogastroduodenoscopy, angiographic embolization or operation to control bleeding within 3 days, and (2) continuous blood transfusion for more than 3 days.

\section{BISHE score}

The BISHE score has been recently developed to predict MT in patients with UGIB and was calculated for all patients. A BISHE score $>7.5$ indicates a high risk of in-hospital mortality(9).

\section{Treatment protocol}


All clinical management decisions were left to the discretion of the main attending physician. Standard management for all patients with UGIB in our ED was administration of an intravenous proton pump inhibitor before endoscopy for non-variceal bleeding. The choice of intermittent or continuous infusion of proton pump inhibitor was left to the discretion of the lead attending clinician. Intravenous terlipressin with an intravenous third-generation cephalosporin was administered before endoscopy for variceal bleeding. Blood transfusion was indicated for patients with a hemoglobin level $<8 \mathrm{~g} / \mathrm{dL}$ or signs of hemodynamic instability despite fluid resuscitation. The decision to administer a transfusion was made by the main attending physician.

\section{Statistical analysis}

Categorical variables are presented as the percentage and continuous variables as the median with the interquartile range. Associated factors were entered as categorical variables to construct a diagnostic scoring system. The cut-off points for all the laboratory variables were determined based on a combination of clinical experience, review of previous reports, and the median values in studies that included MT and non-MT groups(18-20). Univariate and multivariate logistic regression analyses were used in the current study. The initial model inclusion criterion was a p-value of $<0.05$, with predictive factors entered using backward-stepwise selection and retained when the $p$-value was $<0.01$. Assignment of score points was based on the corresponding regression coefficients. By dividing the coefficients by the smallest coefficients in the model and then rounding to the nearest 0.5 , the regression coefficients were transformed to obtain item scores(21).

The risk score for each patient was calculated by totaling the scores for each independent variable. The Hosmer-Lemeshow goodness-of-fit test was used to evaluate the calibration of the model(22). The area under the receiver-operating characteristic (AUROC) curve was calculated to assess the predictive accuracy of the risk score(23). All analyses were conducted using MedCalc Statistical Software version 17.9.2 (MedCalc Software bvba, Ostend, Belgium; http://www.medcalc.org; 2017).

\section{Results}

During the study period (2016-2019), a total of 635 adult patients with UGIB presented to the ED. Seven patients for whom data were incomplete were excluded, leaving 628 patients with complete data for inclusion in the study. Figure 1 shows the study enrolment process. Sixteen $(2.5 \%)$ of the 628 patients died within 30 days and 114 (18.2\%) had further bleeding. Moreover, 110 (17.5\%) required MT; 7 (6.4\%) of these patients died within 30 days. The demographic characteristics and comorbidities of the study participants are shown in Table 1.

Table 2 summarizes the factors associated with MT in multivariate logistic analysis and the corresponding scoring system. The multivariate analysis revealed that liver cirrhosis (odds ratio [OR] 1.81; 
95\% confidence interval [Cl] 1.07-3.08), an international normalized ratio >1.5 (OR 2.08; 95\% Cl 1.11$3.90)$, and hemoglobin $<8 \mathrm{~g} / \mathrm{dL}(\mathrm{OR} 12.79 ; 95 \% \mathrm{Cl} 7.75-21.12)$ were associated with MT.

The score was developed by dividing the regression coefficients of each independent risk factor by the smallest coefficient in the final logistic model and then rounding (Table 2). The performance of the final model was very good (Hosmer-Lemeshow goodness of fit test, $p=0.44)(21)$ and discriminated well between patients with and without MT. The AUROC for the study participants was $0.82(95 \% \mathrm{Cl} 0.78-$ 0.85) (22) (Figure 2).

Table 3 summarizes the sensitivity, specificity, positive predictive value, and negative predictive value by different new score cut-off values.

In this study, the new score (AUROC $0.82,95 \% \mathrm{Cl} 0.78-0.85$ ) performed better than the BISHE score (AUROC $0.73,95 \% \mathrm{Cl} 0.69-0.76, \mathrm{p}=0.004$ ) for prediction of the need for MT (24) (Figure 3).

\section{Discussion}

The main finding of this study was that the new scoring system for MT in patients with UGIB was more accurate and easier to use than the BISHE score.

There is scant information in the literature on validated predictive scoring systems that can be used to assess the need for MT and guide activation of an MT protocol in patients with non-traumatic bleeding. Gastrointestinal bleeding accounts for about $14 \%$ of cases of major bleeding requiring $\operatorname{MT}(25,26)$. Although most cases of UGIB are minor and not associated with hemodynamic instability, transfusions are considered life-saving in the event of massive exsanguinating hemorrhage. Nevertheless, there is no consensus regarding the transfusion strategy for major and minor bleeding in $\operatorname{UGIB}(27,28)$. A restrictive transfusion strategy at lower transfusion thresholds was associated with lower 30-day mortality and further bleeding in patients who had UGIB without massive exsanguinating bleeding(20). However, neither optimal criteria for initiation of transfusion or a transfusion protocol for patients with UGIB and massive hemorrhage have been established. More research concerning transfusion strategies in patients with UGIB was recommended by a recent international consensus conference on blood management(29).

Mortality rates of $20 \%$ and $26 \%$ have been reported for patients requiring $\operatorname{MT}(7,25)$. In our study, about $17.5 \%$ of patients with UGIB required MT, which was associated with a $6.4 \%$ mortality rate. One recent study reported a mortality rate of $6 \%$ in patients with UGIB requiring MT(30), which is consistent with our finding.

Early identification and activation of the protocol is essential to improve outcomes in patients with massive bleeding requiring MT. Several scoring systems that predict the need for MT with high accuracy in trauma patients have been established in recent years $(13,14)$.

Nevertheless, the components of these scoring systems are mainly based on the mechanisms of trauma so are not fully applicable to patients with UGIB. One recent study compared the ability of three scoring 
systems to predict MT in patients with UGIB and showed that the MEW score performed better than the other two scoring systems with an AUROC of $0.77(30)$. The BISHE score is a recently developed scoring system for prediction of the need for MT in patients with UGIB and has shown good performance (AUROC 0.82)(9). In our study, the new scoring system was found to have significantly higher predictive accuracy than the BISHE score (AUROC 0.82 vs 0.73 , p $<0.05$; Fig. 2) and is easy to use. To our knowledge, ours is the first prospective study to validate the BISHE score. This new scoring system can be used to evaluate the need for MT and facilitate initiation of the MT protocol in patients with UGIB.

This study has several potential limitations, the main one being its single-center design. Further clinical experience and external validation of this scoring system will be required to assess its value in the management of individual patients.

\section{Conclusions}

This study introduces a risk scoring system for prediction of the need for MT in patients with UGIB that was developed and validated using a large clinical database. The main value of our findings is that they can be used to enhance clinical decision-making and activation of an MT protocol in patients most likely to benefit. Further research is recommended to investigate the effects of this new scoring system on activation of an MT protocol and clinical outcomes.

\section{List Of Abbreviations}

UGIB: upper gastrointestinal bleeding, AUROC: area under the receiver-operating characteristic, MT: massive transfusion, ED: emergency department, OR: odds ratio, Cl: confidence interval

\section{Declarations}

\section{Ethical Approval and Consent to participate:}

The study was approved by Chang Gung Medical Foundation Institutional Review Board (IRB No: 201600157B0). Informed consent was obtained from all study participants.

\section{Consent for publication}

Not applicable

\section{Availability of data and materials}

The datasets used and analysed during the current study are available from the corresponding author on reasonable request.

\section{Competing interests}

There is no conflict of interest in this study

\section{Funding}


This research was supported by Chang Gung Memorial Hospital, Chiayi under Contract No. CMRPG6F0371.

\section{Authors' contributions}

YCC, KHW, and MSH conceived the study, designed the method. YCC, KHW, and MSH supervised the conduct of the data collection. YCC, KHW, and MSH undertook recruitment of participating centres and patients and managed the data, including quality control. YCC, KHW, and MSH provided statistical advice on study design and analysed the data; YCC chaired the data oversight committee. YCC and MSH drafted the manuscript, and all authors contributed substantially to its revision. MSH takes responsibility for the paper as a whole.

\section{Acknowledgements}

We acknowledge Chang Gung Memorial Hospital, Chiayi for financially supporting this study.

\section{References}

1. Shortt J, Polizzotto MN, Waters N, Borosak M, Moran M, Comande M, et al. Assessment of the urgency and deferability of transfusion to inform emergency blood planning and triage: the Bloodhound prospective audit of red blood cell use. Transfusion. 2009;49(11):2296-303.

2. Subramaniam K, Spilsbury K, Ayonrinde OT, Latchmiah F, Mukhtar SA, Semmens JB, et al. Red blood cell transfusion is associated with further bleeding and fresh-frozen plasma with mortality in nonvariceal upper gastrointestinal bleeding. Transfusion. 2016;56(4):816-26.

3. Abd Elrazek AE, Mahfouz H, Elazeem KA, Fakhry M, Elrazek EA, Foad M, et al. The value of U/S to determine priority for upper gastrointestinal endoscopy in emergency room. Medicine (Baltimore). 2015;94(49):e2241.

4. Sanders DS, Perry MJ, Jones SG, McFarlane E, Johnson AG, Gleeson DC, et al. Effectiveness of an upper-gastrointestinal haemorrhage unit: a prospective analysis of 900 consecutive cases using the Rockall score as a method of risk standardisation. Eur J Gastroenterol Hepatol. 2004;16(5):487-94.

5. Hardy JF. Current status of transfusion triggers for red blood cell concentrates. Transfus Apher Sci. 2004;31(1):55-66.

6. Hardy JF, De Moerloose P, Samama M, Groupe d'interet en Hemostase P. Massive transfusion and coagulopathy: pathophysiology and implications for clinical management. Can J Anaesth. 2004;51(4):293-310.

7. Halmin M, Chiesa F, Vasan SK, Wikman A, Norda R, Rostgaard K, et al. Epidemiology of massive transfusion: a binational study from Sweden and Denmark. Crit Care Med. 2016;44(3):468-77.

8. Fleischer D. Etiology and prevalence of severe persistent upper gastrointestinal bleeding. Gastroenterology. 1983;84(3):538-43.

9. Chen YC, Chuang CJ, Hsiao KY, Lin LC, Hung MS, Chen HW, et al. Massive transfusion in upper gastrointestinal bleeding: a new scoring system. Ann Med. 2019;51(3-4):224-31. 
10. Holcomb JB, del Junco DJ, Fox EE, Wade CE, Cohen MJ, Schreiber MA, et al. The prospective, observational, multicenter, major trauma transfusion (PROMMTT) study: comparative effectiveness of a time-varying treatment with competing risks. JAMA Surg. 2013;148(2):127-36.

11. Callum JL, Nascimento B, Alam A. Massive haemorrhage protocol: what's the best protocol? ISBT Science Series. 2016;11(S1):297-306.

12. McQuilten ZK, Crighton G, Brunskill S, Morison JK, Richter TH, Waters N, et al. Optimal dose, timing and ratio of blood products in massive transfusion: results from a systematic review. Transfus Med Rev. 2018;32(1):6-15.

13. Ogura T, Lefor AK, Masuda M, Kushimoto S. Modified traumatic bleeding severity score: early determination of the need for massive transfusion. Am J Emerg Med. 2016;34(6):1097-101.

14. Nunez TC, Voskresensky IV, Dossett LA, Shinall R, Dutton WD, Cotton BA. Early prediction of massive transfusion in trauma: simple as ABC (assessment of blood consumption)? J Trauma. 2009;66(2):346-52.

15. Maegele M, Lefering $R$, Wafaisade A, Theodorou $P$, Wutzler $S$, Fischer $P$, et al. Revalidation and update of the TASH-Score: a scoring system to predict the probability for massive transfusion as a surrogate for life-threatening haemorrhage after severe injury. Vox Sanguinis. 2011;100(2):231-8.

16. Savage SA, Sumislawski JJ, Zarzaur BL, Dutton WP, Croce MA, Fabian TC. The new metric to define large-volume hemorrhage: results of a prospective study of the critical administration threshold. J Trauma Acute Care Surg. 2015;78(2):224-9; discussion 9-30.

17. Meyer DE, Cotton BA, Fox EE, Stein D, Holcomb JB, Cohen M, et al. A comparison of resuscitation intensity and critical administration threshold in predicting early mortality among bleeding patients: A multicenter validation in 680 major transfusion patients. J Trauma Acute Care Surg. 2018;85(4):691-6.

18. Blatchford O, Murray WR, Blatchford M. A risk score to predict need for treatment for uppergastrointestinal haemorrhage. Lancet. 2000;356(9238):1318-21.

19. Villanueva C, Colomo A, Bosch A, Concepcion M, Hernandez-Gea V, Aracil C, et al. Transfusion strategies for acute upper gastrointestinal bleeding. N Engl J Med. 2013;368(1):11-21.

20. Saltzman JR, Tabak YP, Hyett BH, Sun X, Travis AC, Johannes RS. A simple risk score accurately predicts in-hospital mortality, length of stay, and cost in acute upper GI bleeding. Gastrointest Endosc. 2011;74(6):1215-24.

21. Chen L, Magliano DJ, Balkau B, Colagiuri S, Zimmet PZ, Tonkin AM, et al. AUSDRISK: an Australian Type 2 Diabetes Risk Assessment Tool based on demographic, lifestyle and simple anthropometric measures. Med J Aust. 2010;192(4):197-202.

22. Lemeshow S, Hosmer DW, Jr. A review of goodness of fit statistics for use in the development of logistic regression models. Am J Epidemiol. 1982;115(1):92-106.

23. Hanley JA, McNeil BJ. A method of comparing the areas under receiver operating characteristic curves derived from the same cases. Radiology. 1983;148(3):839-43. 
24. DeLong ER, DeLong DM, Clarke-Pearson DL. Comparing the areas under two or more correlated receiver operating characteristic curves: a nonparametric approach. Biometrics. 1988;44(3):837-45.

25. Ruseckaite R, McQuilten ZK, Oldroyd JC, Richter TH, Cameron PA, Isbister JP, et al. Descriptive characteristics and in-hospital mortality of critically bleeding patients requiring massive transfusion: results from the Australian and New Zealand Massive Transfusion Registry. Vox Sanguinis. 2017;112(3):240-8.

26. Ket SN, Sparrow RL, McQuilten ZK, Tacey M, Gibson PR, Brown GJ, et al. Clinical coding data algorithm to categorize type of gastrointestinal bleeding as a primary reason for massive transfusion: results from the Australian and New Zealand Massive Transfusion Registry. Vox Sanguinis. 2019;114(8):853-60.

27. Barkun AN, Bardou M, Kuipers EJ, Sung J, Hunt RH, Martel M, et al. International consensus recommendations on the management of patients with nonvariceal upper gastrointestinal bleeding. Ann Intern Med. 2010;152(2):101-13.

28. Duggan JM. Gastrointestinal hemorrhage: should we transfuse less? Dig Dis Sci. 2009;54(8):1662-6.

29. Mueller MM, Van Remoortel H, Meybohm P, Aranko K, Aubron C, Burger R, et al. Patient Blood Management: Recommendations From the 2018 Frankfurt Consensus Conference. JAMA. 2019;321(10):983-97.

30. Lee DH, Lee KM, Lee SM, Lee BK, Cho YS, Choi G, et al. Performance of three scoring systems in predicting massive transfusion in patients with unstable upper gastrointestinal hemorrhage. Yonsei Med J. 2019;60(4):368-74.

\section{Tables}

Table 1 Basic demographics and clinical characteristics of the study participants 


\begin{tabular}{lll} 
& Overall & Massive transfusion \\
\hline & $\mathrm{n}(\%)$ & $\mathrm{n}(\%)$ \\
\hline Total & $628(100)$ & $110(17.5)$ \\
\hline Age $>65$ years & $222(35.4)$ & $37(33.6)$ \\
\hline Male & $500(79.6)$ & $89(80.9)$ \\
\hline Medical history & & \\
\hline Ischemic heart disease & $37(5.9)$ & $5(4.5)$ \\
\hline COPD & $8(1.3)$ & $0(0)$ \\
\hline Cerebrovascular accident & $4(0.6)$ & $0(0)$ \\
\hline Rheumatoid disease & $20(3.2)$ & $5(4.5)$ \\
\hline Severe renal disease & $35(5.6)$ & $8(7.3)$ \\
\hline Malignancy & $59(9.4)$ & $13(11.8)$ \\
\hline Chronic hepatitis B & $38(6.1)$ & $7(6.4)$ \\
\hline Chronic hepatitis C & $113(18.0)$ & $18(16.4)$ \\
\hline Liver cirrhosis & $163(26.0)$ & $40(36.4)$ \\
\hline History of UGI bleeding & $154(24.5)$ & $31(28.2)$ \\
\hline Endoscopic finding & & \\
\hline Variceal bleeding & $240(38.2)$ & $59(53.6)$ \\
\hline Non-variceal bleeding & $388(61.8)$ & $51(46.4)$ \\
\hline Laboratory data & & \\
\hline Band form >0 & $57(9.1)$ & $14(12.7)$ \\
\hline Hb <8.0 g/dL & $184(29.3)$ & $83(75.5)$ \\
\hline INR >1.5 & $77(12.3)$ & $25(22.7)$ \\
\hline Treatment & & \\
\hline Endoscopic therapy & $213(33.9)$ & $49(44.5)$ \\
\hline Conditions & & \\
\hline Shock & & \\
\hline BISHE score $>7.5$ & $(38.2)$ & $57.8)$ \\
\hline
\end{tabular}


COPD, chronic obstructive pulmonary disease; Child A, B, and C, Child-Pugh classification A, B, and C (A denotes good hepatic function, $B$ denotes intermediate hepatic function, and $C$ poor function); Band form $>0$, presence of band form cells among white blood cells; Hb, hemoglobin; INR, international normalized ratio; Shock, pulse $>100$ beats per minute or systolic blood pressure $<100 \mathrm{mmHg}$; daytime, from 8:00 am to $5: 00 \mathrm{pm}$; weekend, Saturday and Sunday

Table 2 Factors associated with massive transfusion in final multivariate logistic analysis

\begin{tabular}{llllll} 
& Odds ratio & $95 \% \mathrm{Cl}$ & p-value & coefficient & Score \\
\hline Liver cirrhosis & 1.81 & $1.07-3.08$ & 0.028 & 0.60 & 1 \\
\hline INR $>1.5$ & 2.08 & $1.11-3.90$ & 0.022 & 0.73 & 1 \\
\hline $\mathbf{H b}<\mathbf{8 . 0} \mathbf{g} / \mathbf{d L}$ & 12.79 & $7.75-21.12$ & $<0.001$ & 2.55 & 4
\end{tabular}

$\mathrm{Hb}$, hemoglobin;INR, international normalized ratio. coefficient, the regression coefficient in the multivariate logistic regression model

Table 3 The sensitivity, specificity, positive predictive value, and negative predictive value with the cut-off value in the new scoring system

\begin{tabular}{lllll} 
Cut-off value & Sensitivity & Specificity & PPV & NPV \\
\hline$>0$ & 89.1 & 56.8 & 30.4 & 96.1 \\
\hline$>1$ & 78.2 & 77.8 & 42.8 & 94.4 \\
\hline$>\mathbf{2}$ & 74.6 & 80.7 & 45.1 & 93.7 \\
\hline$>4$ & 33.6 & 93.6 & 52.9 & 86.9 \\
\hline$>5$ & 7.3 & 99.4 & 72.7 & 83.5
\end{tabular}

NPV, negative predictive value; PPV, positive predictive value

\section{Figures}


All adult patients with acute upper gastrointestinal bleeding presenting to the emergency department between July 1, 2016 and June 30, 2019 ( $\mathrm{n}=635)$

Individuals without complete data were excluded

$(n=7)$

All adult patients with acute upper gastrointestinal bleeding presenting at emergency department between July 1,2016 and June 30,2019 with complete data $(\mathrm{n}=628)$

\section{Figure 1}

Flow chart showing the process used to enroll the study participants. 


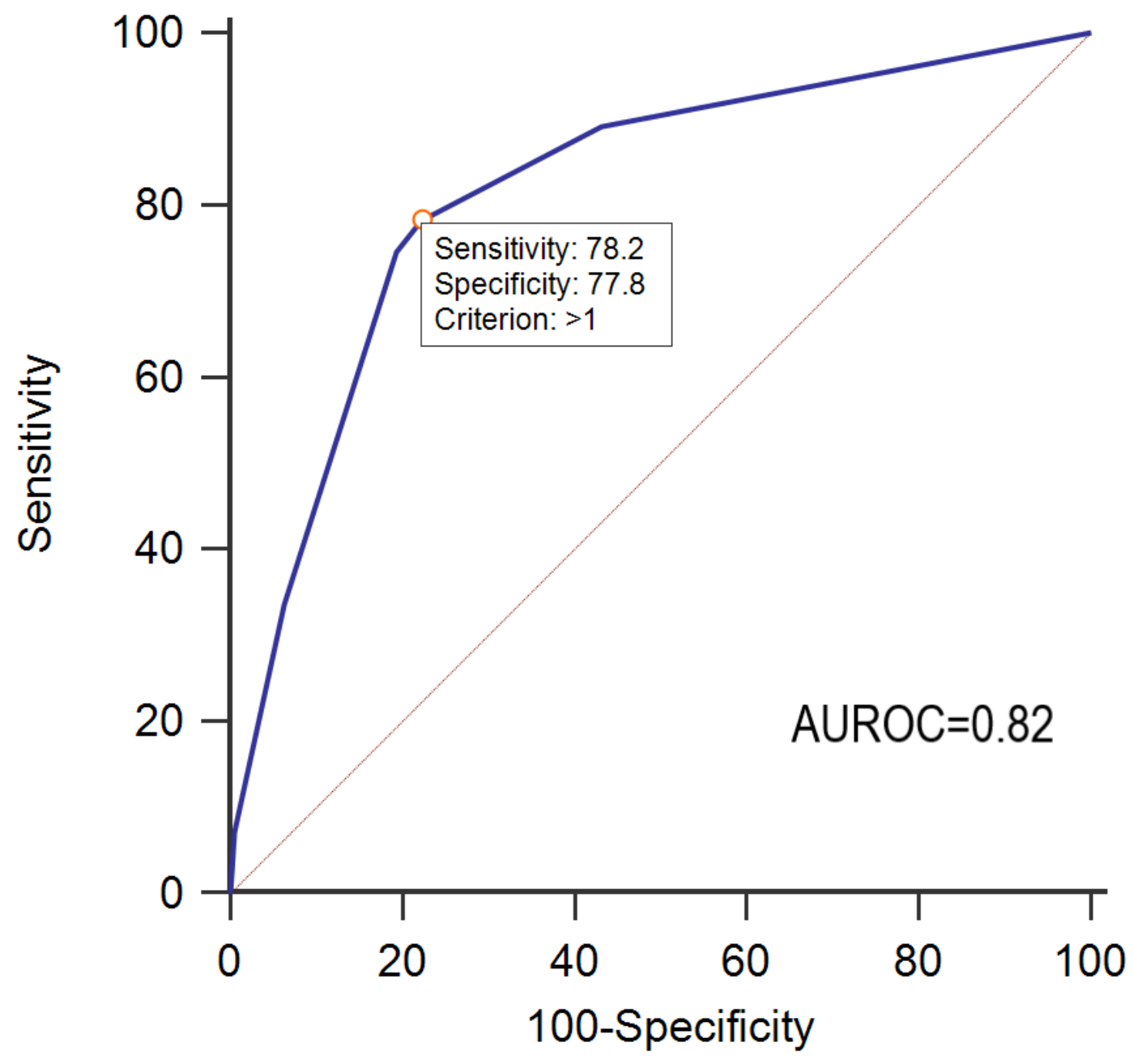

Figure 2

Area under the receiver-operating characteristic curve (AUROC) for the new scoring system. 


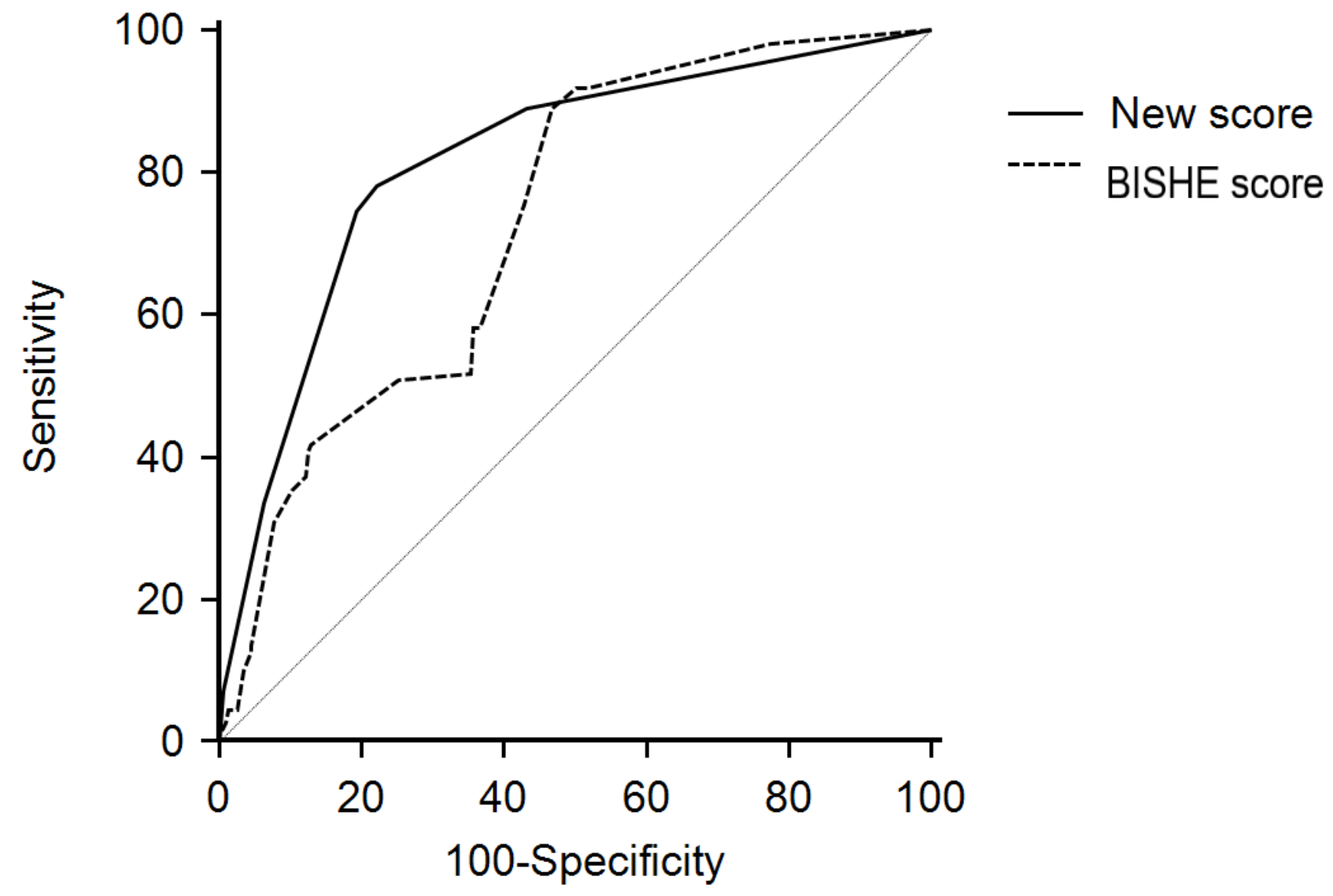

Figure 3

Comparison of the BISHE score and the new score for ability to predict the need for massive transfusion by the area under the receiver-operating characteristic curve (AUROC). 